\title{
ANSIEDADE NA ADOLESCÊNCIA: o ensino médio integrado em foco
}

\author{
Cybele Maria dos Santos Martins ${ }^{1}$ \\ Neide de Brito Cunha ${ }^{2}$
}

\section{RESUMO}

A ansiedade pode se tornar prejudicial quando extrema, podendo acometer até $20 \%$ dos adolescentes. Assim, este estudo objetivou verificar a frequência dos sintomas ansiosos de estudantes de três Cursos Integrados ao Ensino Médio de uma instituição pública de ensino de uma cidade sul mineira e averiguar possíveis diferenças nos níveis de ansiedade entre os sexos, os anos escolares, as idades e os cursos. Participaram do estudo 257 estudantes, de ambos os sexos, do $1^{\circ}$ ao $3^{\circ}$ ano. Os instrumentos utilizados foram: Escala de Ansiedade do Adolescente e de Ansiedade de Auto Avaliação de Zung. Os resultados apontaram níveis de ansiedade normais, o sexo feminino mais ansioso assim como alunos do Curso de Administração com maior nível de ansiedade.

Palavras-chave: Ansiedade. Adolescência. Ensino Médio Integrado.

\section{ANXIETY IN ADOLESCENCE: INTEGRATED HIGH SCHOOL IN FOCUS}

\begin{abstract}
Anxiety can become harmful when extreme, affecting up to $20 \%$ of adolescents, including in the school environment. Thus, this study aimed to verify the frequency of anxious symptoms in students from three Integrated High School Courses at a public educational institution in a city in southern Minas Gerais and to investigate possible differences in anxiety levels between genders, school years, ages and the courses. 257 students participated in the study, of both sexes, from the 1st to the 3rd year. The instruments used were: Adolescent Anxiety Scale and SelfRating Anxiety Scale of Zung. The results showed normal levels of anxiety, female sex more anxious, lack of statistically significant difference between school years and ages, and the Administration Course with a higher level of anxiety.
\end{abstract}

Keywords: Anxiety. Adolescence. Integrated High School.

\section{ANSIEDAD EN LA ADOLESCENCIA: EL BACHILLERATO INTEGRADO EN FOCO}

\footnotetext{
${ }^{1}$ IFSULDEMINAS. E-mail: cybele.martins@yahoo.com.br

${ }^{2}$ Centro Paula Souza - Unidade de Pós-graduação, Extensão e Pesquisa. E-mail: neidedebritocunha@ gmail.com
} 


\section{RESUMEN}

La ansiedad puede volverse dañina cuando extrema, afectando hasta al 20\% de los adolescentes. Así, este estudio tuvo como objetivo verificar la frecuencia de síntomas ansiosos de estudiantes de tres Cursos Integrados de Bachillerato en una institución educativa pública en una ciudad del sur de Minas Gerais e investigar posibles diferencias en los niveles de ansiedad entre géneros, años escolares, edades y los cursos. Participaron 257 estudiantes, de ambos sexos, de $1^{\mathrm{o}}$ a $3^{\mathrm{o}}$ curso. Los instrumentos utilizados fueron: Escala de ansiedad adolescente y Escala de ansiedad de autoevaluación de Zung. Los resultados mostraron niveles normales de ansiedad, el sexo femenino más ansioso, así como los estudiantes del Curso de Administración con mayor nivel de ansiedad.

Palabras clave: Ansiedad. Adolescencia. Bachillerato integrado.

\section{INTRODUÇÃO}

As definições de ansiedade são diversas, assim como as causas de sua ocorrência. Davidoff (2001, p. 390) descreve a ansiedade "como uma emoção caracterizada por sentimentos de antecipação de perigo, tensão e sofrimento e por tendências de esquiva e fuga". Para May (1980), ela apresenta, como característica, uma apreensão sentida pelo indivíduo devido a alguma ameaça àquilo que ele considera essencial à sua personalidade. Freud (1996) considera que a ansiedade constitui uma resposta do ego diante de uma situação de risco com o objetivo de se autopreservar.

Enquanto resposta natural do ser humano ao meio em que vive, a ansiedade é experimentada por todas as pessoas. Ela é um sinal de alerta, muitas vezes indispensável em sua função de prevenir o indivíduo contra possíveis perigos à sua integridade física ou situações emocionalmente dolorosas, asseveram Sadock e Sadock (2007). Brandão (2001) conclui que a ansiedade contém um importante significado adaptativo e evolutivo.

Contudo, quando as reações da ansiedade passam a ser extremas, seja em intensidade ou em frequência, ela deixa de ser considerada normal e benéfica e passa a se tornar patológica, embora seja difícil estabelecer a fronteira entre os dois casos. Os sintomas fisiológicos e psicológicos da ansiedade, que são uma reação ao processamento de estímulos externos, considerados como ameaça pelo sistema nervoso central, são basicamente os mesmos, em ambas as situações (DAVIDOFF, 2001; DUMAS, 2011). 
Embora possam variar de um indivíduo para o outro, as manifestações periféricas de ansiedade podem compreender: diarreia, suor excessivo, vertigem, perda dos sentidos, tremores, irritação gástrica, alteração na frequência urinária, palpitações, agitação, dentre outros. Os sintomas de ordem afetiva e comportamental também podem incluir excesso de vigilância, apreensão, insegurança, irritabilidade, sentimento de desamparo, nervosismo etc. (HERDMAN, 2015; SADOCK; SADOCK, 2007).

Nesse sentido, é necessário o diagnóstico clínico para diferenciar a ansiedade normal dos transtornos de ansiedade. Cada um dos transtornos de ansiedade só pode ser diagnosticado como tal "quando os sintomas não são consequência dos efeitos fisiológicos do uso de uma substância/medicamento ou de outra condição médica ou não são mais bem explicados por outro transtorno mental" (APA, 2014, p. 189). Dalgalarrondo (2008) complementa, afirmando que para o diagnóstico de uma síndrome ansiosa é necessário verificar a extensão do sofrimento e do comprometimento dos sintomas nas esferas social e ocupacional da pessoa.

Em termos estatísticos, os transtornos de ansiedade, bem como os transtornos mentais em geral, possuem alta prevalência. Estudos realizados no Brasil apontam que uma em cada cinco pessoas necessita de atenção referente à saúde mental no período de um ano (MARI; JORGE, 2005; RIBEIRO; POÇO, 2007). Apontam também que as mulheres são as mais acometidas pelos transtornos mentais, principalmente pelos transtornos de ansiedade (ALMEIDA-FILHO et al., 1997; APA, 2014; MARI; JORGE, 2005; MARSH; GRAHAM, 2005; OMS, 2017; RIBEIRO; POÇO, 2007; SADOCK; SADOCK, 2007). Dados recentes da Organização Mundial da Saúde apontaram que 9,3\%, ou seja, 18.657.943 pessoas que vivem no país apresentam transtornos de ansiedade, dados que conferiram ao Brasil o primeiro lugar no rol dos países mais ansioso do mundo (OMS, 2017).

Com relação ao público adolescente, estima-se que metade das doenças mentais se inicia por volta dos 14 anos de idade, e geralmente não é diagnosticada e nem tratada, apesar da grande presença de comorbidades, sendo que, segundo a Organização Mundial da Saúde (OMS), um em cada cinco adolescentes sofre de problemas relacionados à saúde mental. Assim, os transtornos de ansiedade estão entre as psicopatologias mais frequentes da infância e da adolescência, uma prevalência que pode variar de 6 a 20\% deste público (DUMAS, 2011; RIBEIRO; POÇO, 2007). 
Visando levantar pesquisas sobre a saúde mental do adolescente referente à ansiedade, foi realizada uma busca inicial na base de dados da Coordenação de Aperfeiçoamento de Pessoal de Nível Superior (CAPES), Periódicos CAPES, no período de 2014 a 2019, com os seguintes pares de descritores: "ansiedade e adolescência"; "ansiedade e ensino médio" e “ansiedade e ensino médio integrado". Para o primeiro par de descritores foram encontradas 280 pesquisas; para o segundo, 347 e o terceiro par, 23 pesquisas.

A partir desses primeiros resultados, alguns critérios de exclusão foram traçados para a futura análise dos trabalhos a serem levantados, como: pesquisas que não englobassem o período da adolescência; que não abordassem a ansiedade dentre os transtornos mentais pesquisados; que não fossem escritas em português; que objetivassem a validação de escalas psicométricas; e pesquisas que correlacionassem outros temas à ansiedade (doenças, vulnerabilidade social, questões de gênero, uso de medicamentos etc.). Após a utilização desses critérios de exclusão, as 2 pesquisas encontradas se relacionavam apenas ao primeiro e ao segundo par de descritores e novas buscas foram realizadas (no Google Acadêmico, na Scientific Electronic Library Online-SciELO, dentre outros). Contudo, foi ratificada a escassez de pesquisas referentes aos temas pesquisados, sendo eleitos, desta vez, 7 estudos, mas nenhum que abordasse o tema referente à ansiedade e o ensino médio integrado, foco deste estudo.

Dentre as 7 pesquisas, quatro merecem destaque: Thiengo, Cavalcante e Lovisi (2014) demostraram que a prevalência dos transtornos de ansiedade nos adolescentes variou entre 3,3 a 32,3\%. A pesquisa de Andrade, Souza e Castro (2016), com 55 estudantes do último ano do Ensino Médio, objetivou relacionar os exames vestibulares a manifestações psicossomáticas relativas à ansiedade e ao estresse de estudantes. Os resultados apontaram que 45,5\% dos alunos apresentaram ansiedade elevada na escala IDATE estado e 40\% apresentaram ansiedade elevada na escala IDATE traço. O estudo de Lopes et al. (2016) apontou a prevalência dos Transtornos Mentais Comuns (TMC) na adolescência, ou seja, transtornos caracterizados principalmente por sintomas depressivos e ansiosos, de 30\%. Grolli, Wagner e Dalbosco (2017) verificaram a prevalência de sintomas da depressão e da ansiedade em estudantes concluintes do Ensino Médio - entre as mulheres, 13,3\% pontuaram nível grave de ansiedade, 20\% moderado e 6,7\% leve. Já entre os homens, $10 \%$ apresentaram sintomas moderados e 22,5\% em nível leve. 
Parece ser justificável que o público adolescente seja suscetível aos transtornos mentais e de ansiedade, visto que esta fase se caracteriza por diversas transformações, tanto físicas como psicológicas. Há mudanças que exigem uma série de competências socioemocionais para que esse jovem se integre de forma satisfatória ao mundo. Por definição, a adolescência se caracteriza por ser uma fase de crise, transição, adaptação e ajustamento; um estágio em que as mudanças biológicas demandam obrigatoriamente buscas, desencontros, reflexão, abandonos, escolhas e construções. Quando acometidos na adolescência (ou infância), os transtornos mentais podem ser crônicos e trazerem sérios prejuízos na idade adulta (BOHOSLAVSKY, 1993; BRESSAN et al., 2014; DUMAS, 2011; RAPPAPORT, 1993).

Por esse motivo, o ambiente escolar, comum à grande parte do público adolescente, tem se tornado fonte de observação no que se refere à saúde mental, já que é um meio onde as falhas adaptativas causadas pelos transtornos mentais geralmente são mais evidentes. Esses transtornos podem trazer comportamentos como agressividade, irritabilidade, tendência ao isolamento, além de prejudicar o rendimento acadêmico e contribuir para as ausências às aulas e para a evasão escolar (DUMAS, 2011; FLEITLICH-BILYK et al., 2014; VIEIRA et al., 2014).

No caso dos transtornos de ansiedade, sua influência no contexto escolar tem sido foco de vários estudos, apontam Boruchovitch e Costa (2001). Para as autoras, tudo que envolve o ambiente escolar pode ser ansiogênico - disciplinas, professores, colegas etc. - porém, a ansiedade em situação de avaliação tem sido a mais estudada. Estudantes que sofrem de ansiedade diante de situações de provas e avaliações relatam que "ficam bloqueados e que dá um branco na mente" (DAVIDOFF, 2001, p. 401). Complementando, Sadock e Sadock (2007) esclarecem que a ansiedade patológica causa distorções nos processos de percepção do indivíduo. Quando essas alterações acometem a parte cognitiva, é comum afetarem o processo de aprendizagem, diminuindo a concentração, reduzindo a memória e interferindo na capacidade do indivíduo de fazer correlações.

Embora não tenham sido encontrados estudos acerca da saúde mental correlacionadas ao Ensino Médio Integrado, um sistema de ensino que compreende uma proposta desafiadora, conforme aponta Krawczyk (2012), acredita-se que a adaptação e o envolvimento dos estudantes sejam mais prementes do que no Ensino Médio regular, o que poderia agravar problemas de ordem mental. Esse sistema se caracteriza por oferecer seus componentes 
curriculares de forma conjunta (os do ensino propedêutico e os do ensino técnico), ou seja, de forma integrada e interdependente. Ele se define por "integrar teoria e prática, trabalho manual e intelectual, cultura técnica e cultura geral [...]" (KRAWCZYK, 2012, s/p), e suas demandas, por consequência, têm maior complexidade.

Nesse contexto apresentado, a presente pesquisa objetivou verificar a frequência dos sintomas ansiosos de estudantes de três Cursos Integrados ao Ensino Médio de uma instituição pública de ensino de uma cidade do sul de Minas Gerais, e averiguar a existência de possíveis diferenças nos níveis de ansiedade entre os sexos, os anos escolares, as idades e os cursos dos participantes. Os cursos pesquisados - Curso Técnico em Administração, Curso Técnico em Edificações e Curso Técnico em Informática - possuem 2.600 horas de Base Nacional Comum e Parte Diversificada e 120 horas de estágio obrigatório. Com relação ao ensino profissional, o curso de Administração possui 1.000 horas, enquanto os demais possuem 1.200 horas. (IFSULDEMINAS, 2015a; IFSULDEMINAS, 2015b; IFSULDEMINAS, 2016).

\section{MÉTODO}

\section{Participantes}

A amostra contou com 257 estudantes, de ambos os sexos, dos três Cursos Técnicos Integrados ao Ensino Médio de uma instituição pública de ensino de uma cidade do sul de Minas Gerais no ano de 2019, do primeiro ao terceiro ano, totalizando 9 turmas: Curso Técnico em Administração (33,46\% ou 86 estudantes), Curso Técnico em Edificações (86 estudantes ou 33,46\%), e Curso Técnico em Informática (85 estudantes ou 33,08\% da amostra). Dentre os participantes, 59,53\% eram do sexo feminino $(\mathrm{n}=153)$, e $104(40,47 \%)$, do sexo masculino. A faixa etária da amostra foi de 14 a 18 anos, com $(\mathrm{M}=15,89)$.

\section{Instrumentos}

Para maior amplitude e compreensão dos resultados foram utilizados, como instrumentos de coleta de dados, duas escalas para aferição do nível de ansiedade da amostra: a Escala de Ansiedade do Adolescente - EAA (BATISTA, 2007) e a Escala de Ansiedade de Auto Avaliação de Zung (Self-Rating Anxiety Scale of Zung - SAS) (CRUZ, 2008). 
A EAA, escala autoaplicável, contém 41 itens divididos em 5 fatores: ansiedade cognitivo-institucional (F1), que engloba 12 itens; ansiedade antecipatória ou sensibilidade à ansiedade (F2), que contempla 10 itens; ansiedade no relacionamento afetivo, sexual e social (F3), com 9 itens; ansiedade emocional (F4), com 5 itens; e ansiedade sintomática (F5), também contendo 5 itens. As respostas da escala são do tipo Likert, com três pontos, em que se assinala a frequência dos sintomas em cada frase: sempre $=2$ pontos; às vezes $=1$ ponto; $\mathrm{e}$ nunca $=0$. Desse modo, o escore total pode variar de 0 a 82 pontos. Como não há critérios definidos pelo autor da pontuação nos fatores e no total da escala, optou-se, neste estudo, por usar os quartis. Quartil é a denominação do fracionamento, em 4 partes, do conjunto dos dados. O primeiro quartil é o valor do conjunto que delimita os $25 \%$ menores valores. O segundo quartil separa os $50 \%$ menores dos $50 \%$ maiores valores. O terceiro quartil ou quartil superior é o valor que delimita os $25 \%$ maiores valores.

A SAS, também autoaplicável, contém 20 itens, sendo que as questões de 1 a 5 avaliam o componente cognitivo da ansiedade; as questões de 6 a 9, o componente motor; as questões de 10 a 18 avaliam o componente vegetativo, e os itens 19 e 20 avaliam o componente da ansiedade referente ao Sistema Nervoso Central (SNC). Os itens 5, 9, 13, 17 e 19 possuem caráter negativo para o diagnóstico de ansiedade, ou seja, contemplam a ausência de sintomas ansiosos e sua pontuação deve ser feita de forma invertida. As respostas, também do tipo Likert, de quatro pontos, devem ser assinaladas conforme os sintomas que melhor representem cada respondente: nenhuma ou raras vezes $=1$ ponto; algumas vezes $=2$ pontos; uma boa parte do tempo $=3$ pontos; e a maior parte ou a totalidade do tempo $=4$ pontos. A pontuação da SAS varia de 20 a 80 pontos, devendo ser assim interpretada: de 20 a 44 pontos, ansiedade normal; de 45 a 49 pontos, ansiedade leve a moderada; de 60 a 74 pontos, ansiedade intensa e de 75 a 80 pontos, ansiedade extrema.

\section{Procedimentos}

A pesquisa foi aplicada mediante assinatura do Termo de Assentimento por todos os participantes e do Termo de Consentimento Livre e Esclarecido, no caso dos participantes menores de 18 anos de idade sob o Certificado de Apresentação para Apreciação Ética (CAAE) 04755118.0.0000.5102 e Parecer Consubstanciado do Comitê de Ética em Pesquisa (CEP) da Universidade do Vale do Sapucaí (UNIVÁS), Parecer no 3.345.853. A aplicação dos 
instrumentos foi feita de forma coletiva, em cada turma separadamente, necessitando de cinco dias para o término da aplicação nas nove turmas participantes.

Os resultados levantados foram submetidos à análise estatística utilizando o software Statistical Package for the Social Sciences (SPSS), versão 18.0 for Windows. Os resultados foram comparados pelo teste $t$ de Student e pela Anova.

\section{RESULTADOS E DISCUSSÃO}

Para apresentar os resultados relativos ao primeiro objetivo específico da pesquisa verificar a frequência dos sintomas ansiosos dos participantes a partir da Escala de Ansiedade do Adolescente (EAA) e da Escala de Ansiedade de Auto Avaliação de Zung (SAS) - recorreuse à estatística descritiva para identificar a média, o desvio padrão, mínimo, máximo, os quartis e o total das respostas sobre os níveis da ansiedade da amostra, segundo apontam as Tabelas 1 e 2 , respectivamente.

Tabela 1 - Estatísticas descritivas da ansiedade medida pela EAA

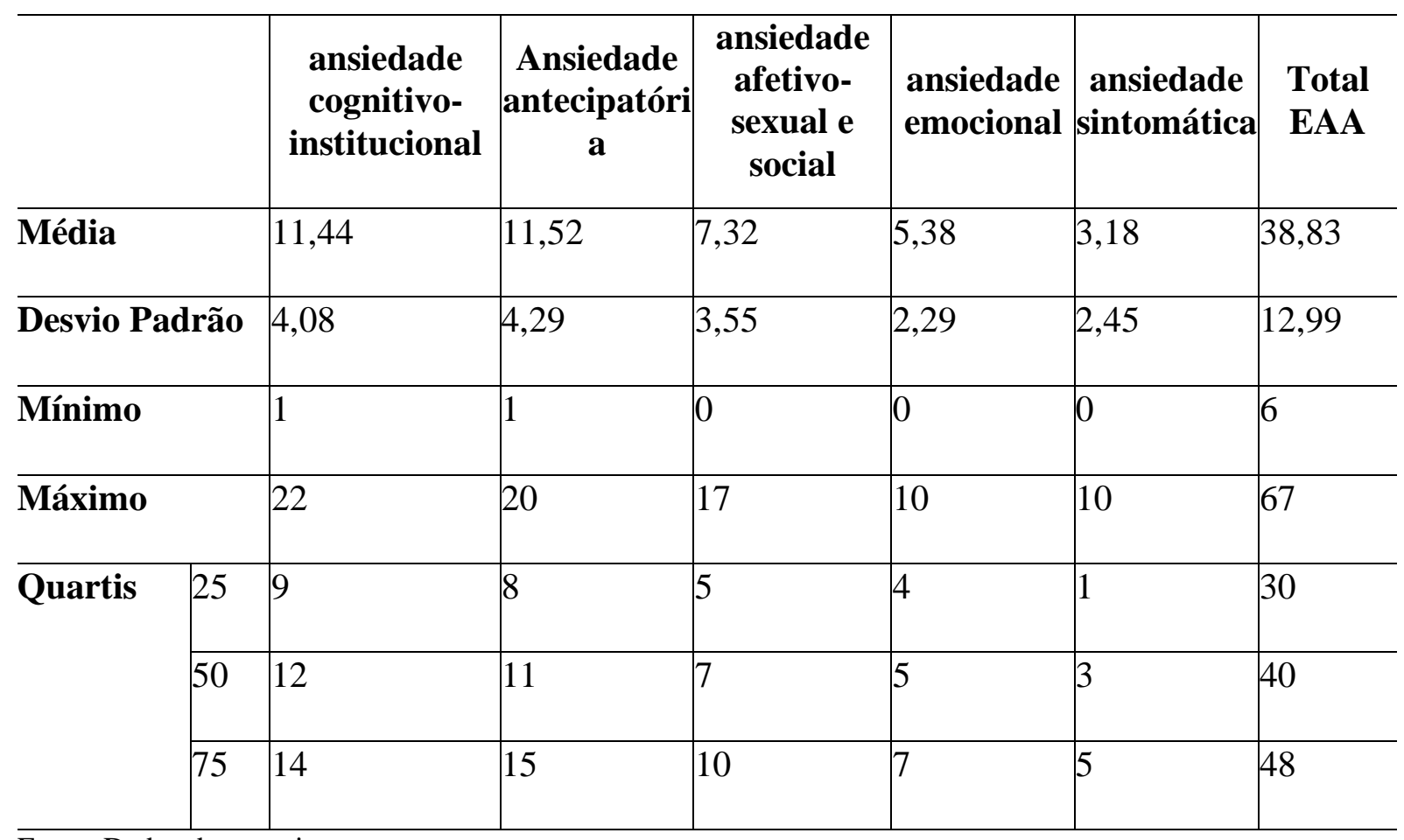

Fonte: Dados da pesquisa.

A Tabela 1 traz que a maior média $(11,52)$ se deu no fator F2 ("ansiedade antecipatória"), que contempla 10 itens da escala, uma média pouco inferior ao F1 ("ansiedade cognitivo-institucional"), que reúne 12 itens do total. A menor média $(3,18)$ ficou com o F5 
("ansiedade sintomática"), representando 5 itens da escala. A média total foi de 38,83, com desvio padrão de 12,99. Considerando que a média total se encontra entre os quartis 25 e 50, os dados demonstram que os níveis dos sintomas ansiosos da amostra podem ser considerados normais pela Escala de Ansiedade do Adolescente (EAA).

Tabela 2 - Estatísticas descritivas da ansiedade medida pela SAS

\begin{tabular}{|c|c|c|c|c|}
\hline & Média & Mínimo & Máximo & $\begin{array}{l}\text { Desvio } \\
\text { Padrão }\end{array}$ \\
\hline componente cognitivo da ansiedade & 11,57 & 5 & 20 & 3,61 \\
\hline componente motor da ansiedade & 9,49 & 4 & 16 & 2,94 \\
\hline componente vegetativo da ansiedade & 17,26 & 9 & 31 & 4,34 \\
\hline componente referente ao SNC & 4,56 & 2 & 8 & 1,62 \\
\hline Total SAS & 42,88 & 22 & 72 & 10,90 \\
\hline
\end{tabular}

Fonte: Dados da pesquisa.

De acordo com a Tabela 2, o "componente vegetativo" alcançou a maior média $(17,26)$, sendo que este contém o maior número de itens da escala (9, dentre os 20$)$. A menor média $(4,56)$ e menor desvio padrão $(\mathrm{DP}=1,62)$ ficaram com o "componente referente ao SNC", que contém o menor número de itens (somente 2). A média total da ansiedade foi de 42,88, com $\mathrm{DP}=10,90$. Conforme interpretação da Escala de Ansiedade de Auto Avaliação de Zung (SAS), a média obtida se enquadra no escore de 20 a 44 pontos, o que corresponde a um nível de ansiedade considerado normal.

As análises das diferenças entre as médias de cada fator e de cada componente das respectivas escalas, trazidas pelas Tabelas $1 \mathrm{e} 2$, sugerem que elas se devam à proporcionalidade do número de itens a que cada um corresponde; ou seja, quanto maior o número de itens que eles possuem, maior a probabilidade de apresentarem uma média maior. Os resultados das Tabelas 1 e 2, ao apontarem que os níveis de sintomas ansiosos da amostra podem ser considerados normais, não descartam a necessidade de se acreditar que a simples presença dos sintomas já pode ser considerada importante. Haja vista as pesquisas acerca do adoecimento 
mental do público adolescente trazidas, dentre outros, por Thiengo, Cavalcante e Lovisi (2014), Andrade, Souza e Castro (2016), Lopes et al. (2016) e Grolli, Wagner e Dalbosco (2017).

Embora a ansiedade seja considerada uma reação natural do organismo, benéfica e com função adaptativa (SADOCK; SADOCK, 2007; DUMAS, 2011), e que atinge a todos os indivíduos, conforme assevera May (1980), é preciso estar atendo à saúde mental do jovem. Afinal, a ansiedade pode se tornar patológica, caso exceda em frequência e em intensidade, causando reações extremas e acarretando possíveis prejuízos na qualidade de vida do indivíduo como um todo (DUMAS, 2011). E é justamente a fase da adolescência o período mais favorável ao aparecimento dos transtornos mentais em geral (BRESSAN et al., 2014).

Portanto, faz-se necessário que se busque sempre um diagnóstico clínico para diferenciar uma ansiedade normal da patológica. Segundo recomendações de Dumas (2011, p. 397), um diagnóstico dos transtornos de ansiedade deve ser baseado "em várias fontes de informação para captar as diversas manifestações [...]”. Essa preocupação se justifica principalmente devido a informações da Organização Mundial a respeito de o Brasil ser o país mais ansioso do mundo (OMS, 2017).

O segundo objetivo específico deste estudo visou averiguar possíveis diferenças dos níveis de sintomas ansiosos entre os sexos, os anos escolares, as idades e os cursos dos participantes. Os resultados estão dispostos nas Tabelas de número 3 a 7, a seguir.

A Tabela 3 traz os resultados da Escala de Ansiedade do Adolescente (EAA) referentes à variável sexo e os resultados do Teste $t$ de Student, usado para comparar as médias de uma variável entre dois grupos diferentes de indivíduos.

Os resultados da Tabela 3 apontam que houve diferença estatisticamente significativa entre os sexos em todos os fatores da escala e em seu total $(p<0,001)$. O sexo feminino $(\mathrm{n}=153)$ apresentou média de 43,98 e o sexo masculino $(n=104)$ apresentou média de 31,30.

A Tabela 4 traz as estatísticas descritivas e o Teste $t$ de Student para verificação das diferenças relacionadas ao fator sexo apontadas pela Escala de Ansiedade de Auto Avaliação de Zung (SAS). 
Tabela 3 - Estatísticas descritivas e Teste $t$ de Student para comparação entre os sexos de acordo com a EAA

\begin{tabular}{|c|c|c|c|c|c|c|}
\hline & Sexo & $\mathbf{N}$ & Média & $\begin{array}{l}\text { Desvio } \\
\text { Padrão }\end{array}$ & $t$ & $p$ \\
\hline \multirow{2}{*}{$\begin{array}{l}\text { ansiedade cognitivo- } \\
\text { institucional }\end{array}$} & Masculino & 104 & 9,82 & 3,94 & \multirow[b]{2}{*}{$-5,535$} & \multirow[b]{2}{*}{$<0,001$} \\
\hline & Feminino & 153 & 12,54 & 3,81 & & \\
\hline \multirow{2}{*}{$\begin{array}{l}\text { ansiedade } \\
\text { antecipatória }\end{array}$} & Masculino & 104 & 8,55 & 3,74 & \multirow[t]{2}{*}{$-11,114$} & \multirow[t]{2}{*}{$<0,001$} \\
\hline & Feminino & 153 & 13,54 & 3,38 & & \\
\hline \multirow{2}{*}{$\begin{array}{l}\text { ansiedade afetivo- } \\
\text { sexual e social }\end{array}$} & Masculino & 104 & 6,29 & 3,41 & \multirow[t]{2}{*}{$-3,930$} & \multirow[t]{2}{*}{$<0,001$} \\
\hline & Feminino & 153 & 8,02 & 3,49 & & \\
\hline \multirow[t]{2}{*}{ ansiedade emocional } & Masculino & 104 & 4,44 & 2,32 & \multirow[t]{2}{*}{$-5,708$} & \multirow[t]{2}{*}{$<0,001$} \\
\hline & Feminino & 153 & 6,01 & 2,04 & & \\
\hline \multirow[t]{2}{*}{ ansiedade sintomática } & Masculino & 104 & 2,20 & 2,20 & \multirow[t]{2}{*}{$-5,546$} & \multirow[t]{2}{*}{$<0,001$} \\
\hline & Feminino & 153 & 3,84 & 2,39 & & \\
\hline \multirow[t]{2}{*}{ Total EAA } & Masculino & 104 & 31,30 & 11,89 & \multirow[t]{2}{*}{$-8,725$} & \multirow[t]{2}{*}{$<0,001$} \\
\hline & Feminino & 153 & 43,98 & 11,08 & & \\
\hline
\end{tabular}

Fonte: Dados da pesquisa.

Os resultados da SAS, trazidos pela Tabela 4, demonstram que todos os componentes da escala e o seu total também apresentaram diferença estatisticamente significativa entre o sexo masculino e feminino ( $p<0,001)$. O sexo feminino apresentou média de 46,07 , enquanto a média do sexo masculino foi de 38,18 .

Com relação aos resultados das Tabelas 3 e 4, ambas as escalas apontaram que as mulheres são mais ansiosas que os homens. Esse resultado consolida dados da literatura científica e de pesquisas brasileiras e internacionais que apontam que não só os transtornos de ansiedade, mas os transtornos mentais como um todo são mais prevalentes no sexo feminino (ALMEIDA-FILHO et al., 1997; APA, 2014; MARI; JORGE, 2005; MARSH; GRAHAM, 2005; OMS, 2017; RIBEIRO; POÇO, 2007; SADOCK; SADOCK, 2007). 
Tabela 4 - Estatísticas descritivas e Teste $t$ de Student para comparação entre os sexos de acordo com a SAS

\begin{tabular}{|c|c|c|c|c|c|c|}
\hline & Sexo & $\mathbf{N}$ & Média & $\begin{array}{l}\text { Desvio } \\
\text { Padrão }\end{array}$ & $t$ & $p$ \\
\hline \multirow[t]{2}{*}{ componente cognitivo } & Masculino & 104 & 10,09 & 3,29 & \multirow[t]{2}{*}{$-5,748$} & \multirow[t]{2}{*}{$<0,001$} \\
\hline & Feminino & 153 & 12,58 & 3,48 & & \\
\hline \multirow[t]{2}{*}{ componente motor } & Masculino & 104 & 8,08 & 2,66 & \multirow[t]{2}{*}{$-6,911$} & \multirow[t]{2}{*}{$<0,001$} \\
\hline & Feminino & 153 & 10,45 & 2,72 & & \\
\hline \multirow[t]{2}{*}{ componente vegetativo } & Masculino & 104 & 15,86 & 3,69 & \multirow[t]{2}{*}{$-4,419$} & \multirow[t]{2}{*}{$<0,001$} \\
\hline & Feminino & 153 & 18,21 & 4,49 & & \\
\hline \multirow[t]{2}{*}{ componente SNC } & Masculino & 104 & 4,16 & 1,63 & \multirow[t]{2}{*}{$-3,320$} & \multirow[t]{2}{*}{$<0,001$} \\
\hline & Feminino & 153 & 4,84 & 1,57 & & \\
\hline \multirow[t]{2}{*}{ Total SAS } & Masculino & 104 & 38,18 & 9,46 & \multirow[t]{2}{*}{$-6,079$} & \multirow[t]{2}{*}{$<0,001$} \\
\hline & Feminino & 153 & 46,07 & 10,68 & & \\
\hline
\end{tabular}

Fonte: Dados da pesquisa.

Para se verificar os dados do nível de ansiedade dos participantes alusivos aos seus respectivos anos escolares segundo a Escala de Ansiedade do Adolescente (EAA) e a Escala de Ansiedade de Auto Avaliação de Zung (SAS), apresenta-se a Tabela 5.

Os dados da Tabela 5 apontam que tanto na EAA quanto na SAS houve pouca diferença das médias entre os anos escolares da amostra. A maior média da EAA foi dos $3^{\text {os }}$ anos $(39,83)$, seguida pela média dos $2^{\text {os }}$ anos $(38,93)$ e dos $1^{\text {os }}$ anos $(37,98)$. Pela SAS, contata-se que a maior média foi apresentada pelos $2^{\text {os }}$ anos $(43,54)$; nos $3^{\text {os }}$ anos a média foi de 43,16 , e nos $1^{\text {os }}$ anos, 42,15. Dessa forma, verifica-se, pela Tabela 5, que, com exceção do segundo fator (F2) da Escala de Ansiedade do Adolescente (EAA) - "ansiedade antecipatória" ( $p<0,05)$, não houve diferença estatisticamente significativa entre os anos escolares referente aos níveis de ansiedade da amostra. 
Tabela 5 - Análise de Variância para verificar diferença entre as médias da EAA e da SAS por ano escolar

\begin{tabular}{|c|c|c|c|c|c|c|c|c|c|c|c|}
\hline \multirow{2}{*}{$\begin{array}{l}\text { Fatores/ } \\
\text { compone } \\
\text { ntes }\end{array}$} & \multicolumn{3}{|c|}{$1^{\text {os }}$ anos } & \multicolumn{3}{|c|}{$2^{o s}$ anos } & \multicolumn{3}{|c|}{$3^{\text {os }}$ anos } & \multirow[b]{2}{*}{$F$} & \multirow[b]{2}{*}{$p$} \\
\hline & $\mathbf{M}$ & $\mathbf{N}$ & DP & $\mathbf{M}$ & $\mathbf{N}$ & DP & $\mathbf{M}$ & $\mathbf{N}$ & DP & & \\
\hline $\begin{array}{l}\text { cognitivo } \\
- \\
\text { institucio } \\
\text { nal }\end{array}$ & 11,15 & 101 & 4,43 & 11,66 & 80 & 4,05 & 11,58 & 76 & 3,62 & 0,418 & 0,65 \\
\hline $\begin{array}{l}\text { antecipat } \\
\text { ória }\end{array}$ & 10,64 & 101 & 4,15 & 12,29 & 80 & 4,37 & 11,87 & 76 & 4,24 & 3,709 & $\begin{array}{l}<0,0 \\
5\end{array}$ \\
\hline $\begin{array}{l}\text { afetivo- } \\
\text { sexual e } \\
\text { social }\end{array}$ & 7,54 & 101 & 3,57 & 6,86 & 80 & 3,51 & 7,50 & 76 & 3,59 & 0,949 & 0,38 \\
\hline $\begin{array}{l}\text { emociona } \\
\text { l }\end{array}$ & 5,14 & 101 & 2,28 & 5,44 & 80 & 2,50 & 5,63 & 76 & 2,07 & 1,041 & 0,35 \\
\hline $\begin{array}{l}\text { sintomáti } \\
\text { ca }\end{array}$ & 3,51 & 101 & 2,41 & 2,68 & 80 & 2,18 & 3,25 & 76 & 2,68 & 2,708 & 0,06 \\
\hline $\begin{array}{l}\text { Total } \\
\text { EAA }\end{array}$ & 37,98 & 101 & 12,91 & 38,93 & 80 & 13,85 & 39,83 & 76 & 12,23 & 0,43 & 0,64 \\
\hline cognitivo & 11,24 & 101 & 3,58 & 11,86 & 80 & 3,67 & 11,70 & 76 & 3,60 & 0,735 & 0,48 \\
\hline motor & 9,41 & 101 & 2,92 & 9,64 & 80 & 3,04 & 9,45 & 76 & 2,87 & 0,149 & 0,86 \\
\hline $\begin{array}{l}\text { vegetativ } \\
\text { o }\end{array}$ & 16,93 & 101 & 4,26 & 17,46 & 80 & 4,14 & 17,47 & 76 & 4,66 & 0,468 & 0,62 \\
\hline SNC & 4,57 & 101 & 1,54 & 4,57 & 80 & 1,63 & 4,54 & 76 & 1,73 & 0,012 & 0,98 \\
\hline $\begin{array}{l}\text { Total } \\
\text { SAS }\end{array}$ & 42,15 & 101 & 10,51 & 43,54 & 80 & 10,96 & 43,16 & 76 & 11,42 & 0,395 & 0,67 \\
\hline
\end{tabular}

Fonte: Dados da pesquisa.

A Tabela 5 demonstrou que basicamente não foi encontrada diferença estatisticamente significativa entre os $1^{\text {os }}, 2^{\text {os }}$ e $3^{\text {os }}$ anos, tanto pela Escala de Ansiedade do Adolescente (EAA) como pela Escala de Ansiedade de Auto Avaliação de Zung (SAS). Assim, é possível inferir 
que embora os agentes estressores possam ser diferentes de um ano escolar para outro, eles atingem as turmas de forma semelhante.

Para as turmas de $1^{\circ}$ ano, talvez o principal agente estressor seja a entrada em um novo nível de ensino - do Fundamental para o Ensino Médio, além das mudanças sentidas com o início da adolescência. No caso dos $3^{o s}$ anos, é possível que a ansiedade se dê por conta da iminente entrada na vida adulta: as preocupações e inseguranças referentes à escolha profissional, do curso superior e/ou da entrada no mundo do trabalho. Já as turmas de $2^{\circ}$ ano devem sentir como agentes estressores a sua posição entre esses dois pontos: estão ainda sob efeito das crises naturais da adolescência, enquanto já se preocupam com as demandas da vida adulta.

Tabela 6 - Análise de Variância para verificar diferença entre as médias dos fatores e componentes da ansiedade por idade

\begin{tabular}{l|l|l}
\hline & \multicolumn{1}{|c}{ F } & \multicolumn{1}{c}{$p$} \\
\hline ansiedade cognitivo-institucional & 1,570 & 0,183 \\
\hline ansiedade antecipatória & 3,477 & $<0,05$ \\
\hline ansiedade afetivo-sexual e social & 2,495 & 0,05 \\
\hline ansiedade emocional & 1,371 & 0,245 \\
\hline ansiedade sintomática & 0,170 & 0,953 \\
\hline Total EAA & 1,405 & 0,233 \\
\hline componente cognitivo & & 0,212 \\
\hline componente motor & 2,352 & 0,348 \\
\hline componente vegetativo & 1,120 & 0,271 \\
\hline componente SNC & 1,299 & \\
\hline Total SAS & 0,649 & \\
\hline
\end{tabular}

Fonte: Dados da pesquisa. 
Em seguimento à demonstração dos resultados, recorreu-se à análise de Variância ANOVA para se verificar as possíveis diferenças nos níveis de sintomas ansiosos entre participantes com idades diferentes. A Tabela 6 oferece os resultados da Escala de Ansiedade do Adolescente (EAA) e da Escala de Ansiedade de Auto Avaliação de Zung (SAS) por idade.

De acordo com a Tabela 6, houve diferença estatisticamente significativa $(p<0,05)$ referente aos níveis de sintomas ansiosos entre as idades apenas no segundo e terceiro fatores (F2, "ansiedade antecipatória" e F3, "ansiedade afetivo-sexual e social") da EAA e no "componente cognitivo" da SAS.

As explicações sobre as diferenças estatísticas recaírem no F2 e F3 da EAA e no “componente cognitivo" da SAS, conforme mostra a Tabela 6, devem se pautar no fato de eles fazerem alusão às inseguranças e medos muito comuns na adolescência, em suas distintas faixas etárias: o F3 se refere a inseguranças relacionadas à sexualidade e à afetividade, típicas do início da adolescência; o F2 trata de medos relacionados à vida adulta, algo comum em adolescentes mais velhos; e o "componente cognitivo" alude à natureza afetiva da ansiedade, ou seja, a sensações e sentimentos como insegurança, inquietações e medos, conforme as características afetivas da ansiedade descritas por Herdman (2015). Com relação aos medos relacionados à vida adulta, dentre eles o vestibular, por exemplo, a pesquisa de Andrade, Souza e Castro (2016) apontou que o estresse e a ansiedade diante do vestibular apresentaram grande incidência. Pressão familiar e escolar, e a tomada de decisão frente o futuro foram apontados como fatores causais.

Para finalizar os resultados relativos ao segundo e último objetivo específico deste estudo, a Tabela 7 apresenta os dados obtidos pela Escala de Ansiedade do Adolescente (EAA) e pela Escala de Ansiedade de Auto Avaliação de Zung (SAS) sobre as possíveis diferenças nos níveis de sintomas ansiosos entre os cursos dos participantes por meio da análise de Variância ANOVA. 
Tabela 7 - Análise de Variância para verificar diferença entre as médias da EAA e SAS por curso

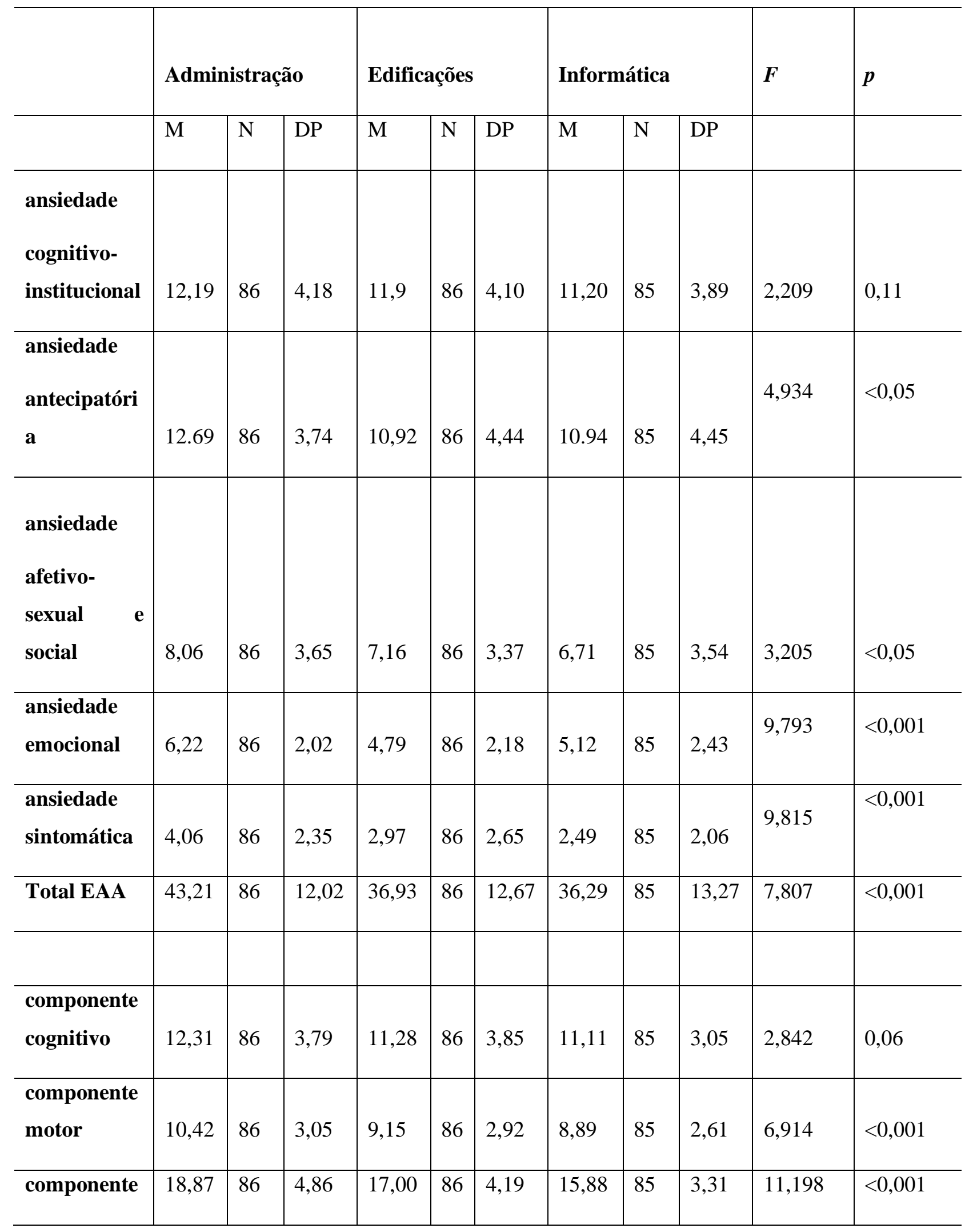




\begin{tabular}{l|l|l|l|l|l|l|l|l|l|l|l}
\hline vegetativo & & & & & & & & & & & \\
\hline $\begin{array}{l}\text { componente } \\
\text { SNC }\end{array}$ & 4,78 & 86 & 1,67 & 4,59 & 86 & 1,70 & 4,32 & 85 & 1,47 & 1,751 & 0,17 \\
\hline Total SAS & 46,38 & 86 & 11,81 & 42,02 & 86 & 11,06 & 40,20 & 85 & 8,75 & 7,648 & $<0,001$ \\
\hline
\end{tabular}

Fonte: Dados da pesquisa.

Pela Tabela 7 nota-se que, com exceção do fator F1 ("ansiedade cognitivoinstitucional"), houve diferença estatisticamente significativa do nível de ansiedade nos demais fatores da EAA. A maior média se deu no Curso Técnico em Administração (43,21), e a menor, no Curso Técnico em Informática $(36,29)$, que apresentou o maior desvio padrão ( $D P=13,27)$. Quanto à SAS, dois dos seus quatro componentes apresentaram diferença estatisticamente significativa ("componente motor" e "componente vegetativo"), assim como o seu total. A maior média $(46,38)$ e o maior desvio padrão $(\mathrm{DP}=11,81)$ se deram no Curso Técnico em Administração. Já a menor média $(40,20)$ e o menor desvio padrão $(\mathrm{DP}=8,75)$ foram vistos no Curso Técnico em Informática.

Os resultados trazidos pela Tabela 7 apontaram que o curso com maior incidência da ansiedade foi o Curso Técnico em Administração, seguido pelo Curso Técnico em Edificações e pelo Curso Técnico em Informática. Embora o curso de Administração apresente menor carga horária que os demais (IFSULDEMINAS, 2015b), fato que poderia supor um resultado diferente do apresentado, há que se levar em conta as individualidades humanas. Assim como existem pessoas mais suscetíveis às pressões do dia a dia do que outras, de acordo com Watson e Clark (1984 apud DAVIDOFF, 2001), pode-se considerar que a amostra do Curso Técnico em Administração congregasse maior quantidade de estudantes mais suscetíveis à ansiedade do que as dos demais cursos.

Conforme afirma Freud (1996, p. 131), “a ansiedade não é assim um assunto tão simples". Ela demonstra, mais do que nunca, sua natureza multifacetada quando se encaram os desafios contemporâneos, sobretudo para aqueles que trabalham com saúde mental. Nesse sentido, estudar a influência do sistema e/ou do ambiente escolar na etiologia da ansiedade, enquanto um dos fatores externos que a compõem, justifica o estudo do tema.

É necessário que as instituições escolares, consideradas pela própria Organização Mundial de Saúde como um dos principais agentes de prevenção e promoção da saúde - mental 
e geral - atuem como agentes redutores de fatores de risco. Quanto ao Ensino Médio Integrado, acredita-se que a presente pesquisa evidenciou a necessidade de estudos mais amplos sobre sua influência perante a saúde mental de seus estudantes.

\section{CONSIDERAÇÕES FINAIS}

Acredita-se que este estudo, embora seja um pequeno recorte empírico, tenha demonstrado que o sofrimento mental e os transtornos de ansiedade na adolescência precisam de atenção. A autora principal, como psicóloga da instituição analisada, recebe muitas queixas dos alunos e foi neste intuito que trouxe, neste trabalho, a subseção sobre a ansiedade no contexto escolar. As autoras consideram que as instituições de ensino, consideradas pela própria Organização Mundial de Saúde como principais agentes para prevenir e promover a saúde mental e geral - necessitam agir como agentes redutores de fatores de risco de seus estudantes, ou seja, como agentes protetores.

Nesse sentido, instala-se o desafio de as instituições educacionais pensarem em iniciativas e intervenções, principalmente pedagógicas, que visem minimizar os impactos negativos sobre a saúde mental de seus estudantes. Sabe-se, porém, que as responsabilidades dessas instituições são apenas parte de uma conjuntura maior, permanece ainda, dentre outros, o viés particular, subjetivo de cada estudante frente aos transtornos de ansiedade e da saúde mental como um todo.

Cabe atentar, então, para os cuidados que se deve tomar ao se conceituar um transtorno mental, diferenciando-o de outras situações ao se fazer seu diagnóstico assertivo e clínico. São precauções que visam à busca do tratamento adequado, obviamente, mas, que antes disto, evitam o sofrimento e o desgaste emocionais oriundos de uma eventual interpretação equivocada dos sintomas. Esse fato parece ser um divisor de águas frente à realidade vivenciada por quem trabalha com a saúde mental, sobretudo dos jovens.

\section{AGRADECIMENTOS}

Apoio à pesquisa do IFSULDEMINAS. 


\section{REFERÊNCIAS}

ALMEIDA-FILHO, N. et al. Brazilian multicentric study of psychiatric morbidity: methodological features and prevalence estimates. British Journal of Psychiatric. London: Royal College of Psychiatrists, v. 171, p. 524-529, 1997. Disponível em: https://www.researchgate.net/publication/13730433. Acesso em: 17 abr. 2019.

AMERICAN PSYCHIATRIC ASSOCIATION. Manual diagnóstico e estatístico de transtornos mentais: DSM-5. Tradução: Maria Inês C. Nascimento. 5. ed. Porto Alegre: Artmed, 2014.

ANDRADE, T. M.; SOUZA, V. N.; CASTRO, N. R. Nível de ansiedade e estresse em adolescentes concluintes do ensino médio. Revista Científica Univiçosa, Viçosa, v. 8, n.1, p. 595-600, jan./dez. 2016. Disponível em:

https://academico.univicosa.com.br/revista/index.php/RevistaSimpac/article/view/706/861. Acesso em: 19 jun. 2019.

BATISTA, M. A. Escala de ansiedade do adolescente - EAA: estudos psicométricos. 2007. Tese (Doutorado em Psicologia) - Universidade São Francisco, Itatiba, 2007.

BOHOSLVASKY, R. Orientação vocacional: a estratégia clínica. 10. ed. São Paulo: Martins Fontes, 1993.

BORUCHOVITCH, E.; COSTA, E. R. da. O impacto da ansiedade no rendimento escolar e na motivação de alunos. In: BORUCHOVITCH, E.; BZUNECK, J. A. (org.). A motivação do aluno: contribuições da psicologia contemporânea. 3. ed. Petrópolis, RJ: Vozes, 2001, p. 134147.

BRANDÃO, M. L. Psicofisiologia: as bases fisiológicas do comportamento. 2. ed. São Paulo: Editora Atheneu, 2001.

BRESSAN, R. A. et al. Promoção da saúde mental e prevenção de transtornos mentais no contexto escolar. In: ESTANISLAU, G. M.; BRESSAN, R. A. (org.). Saúde Mental na escola: o que os educadores devem saber. Porto Alegre: Artmed, 2014, p. 37-47.

CRUZ, M. A. C. da. Ansiedade e bem-estar na transição para o ensino superior: o papel do suporte social. 2008. Dissertação (Mestrado em Psicologia) - Faculdade de Psicologia e de Ciências da Educação da Universidade do Porto, Portugal, 2008. Disponível em: https://repositorio-aberto.up.pt/bitstream/10216/23383/2/29839.pdf. Acesso em: 22 out. 2018.

DALGALARRONDO, P. Psicopatologia e semiologia dos transtornos mentais. 2. ed. Porto Alegre: Artmed, 2008.

DAVIDOFF, L. L. Introdução à Psicologia. Tradução: Lenke Peres. 3. ed. São Paulo: Pearson Makron Books, 2001.

DUMAS, J. E. Psicopatologia da infância e da adolescência. 3. ed. Porto Alegre: Artmed, 2011. 
FLEITLICH-BILYK, B. et al. Saúde e transtornos mentais. In: ESTANISLAU, G. M.; BRESSAN, R. A. (org.). Saúde Mental na escola: o que os educadores devem saber. Porto Alegre: Artmed, 2014, p. 25-36.

FREUD, S. Obras completas de Sigmund Freud: estudo autobiográfico; inibições, sintomas e ansiedade; a questão da análise leiga e outros trabalhos (1925-1926). Volume XX. Tradução: Jayme Salomão. Rio de Janeiro: Imago Editora, 1996.

GROLLI, V.; WAGNER, M. F.; DALBOSCO, S. N. P. Sintomas Depressivos e de Ansiedade em Adolescentes do Ensino Médio. Revista de Psicologia da IMED, Passo Fundo, v. 9, n. 1, p. 87-103, nov. 2017. Disponível em: https://seer.imed.edu.br/index.php/revistapsico/article/view/2123. Acesso em: 20 set. 2018.

HERDMAN, T. H. Enfrentamento/tolerância ao estresse. In: HERDMAN, T. H.; KAMITSURU, S. (org.). Diagnóstico de enfermagem da NANDA: definições e classificação 2015-2017. Tradução: Regina M. Garcez. 10. ed. Porto Alegre: Artmed, 2015. p. 303-347.

INSTITUTO FEDERAL DE EDUCAÇÃO, CIÊNCIA E TECNOLOGIA DO SUL DE MINAS GERAIS (IFSULDEMINAS). Resolução CONSUP no 88, de 17 de dezembro de 2015. Dispõe sobre a aprovação da reformulação e atualização do PPC do Curso Técnico em Informática integrado - Campus Pouso Alegre - IFSULDEMINAS. Pouso Alegre, 2015a. Disponível em: https://portal.ifsuldeminas.edu.br/images/PDFs/Conselho_Superior_/resolucoes/2015/58100/resolucao88.pdf. Acesso em: 14 jun. 2019.

INSTITUTO FEDERAL DE EDUCAÇÃO, CIÊNCIA E TECNOLOGIA DO SUL DE MINAS GERAIS (IFSULDEMINAS). Resolução CONSUP nº 89, de 17 de dezembro de 2015. Dispõe sobre a aprovação da reformulação e atualização do PPC do Curso Técnico em Administração integrado - Campus Pouso Alegre - IFSULDEMINAS. Pouso Alegre, 2015b. Disponível em: https://portal.ifsuldeminas.edu.br/images/PDFs/Conselho_Superior_/resolucoes/2015/58100/resolucao89.pdf. Acesso em: 14 jun. 2019.

INSTITUTO FEDERAL DE EDUCAÇÃO, CIÊNCIA E TECNOLOGIA DO SUL DE MINAS GERAIS (IFSULDEMINAS). Resolução CONSUP n⿳0 63, de 14 de setembro de 2016. Dispõe sobre a Homologação da Resolução "ad referendum" 055/2016 que trata da Criação do Curso Técnico em Edificações Integrado ao Ensino Médio - Campus Pouso Alegre. Pouso Alegre, 2016. Disponível em: https://portal.poa.ifsuldeminas.edu.br/images/2018/Outubro/11/Resolu\%C3\%A7\%C3\%A3o_ edifica\%C3\%A7\%C3\%B5es.pdf. Acesso em: 14 jun. 2019.

KRAWCZYK, N. Novos formatos escolares para novas demandas sociais: o Ensino Médio Integrado. Archivos de Ciencias de la Educación, v. 6, n. 6, 2012. Disponível em: http://www.memoria.fahce.unlp.edu.ar/art_revistas/pr.5928/pr.5928.pdf. Acesso em: 4 jun. 2019.

LOPES, C. S. et al. ERICA: prevalência de transtornos mentais comuns em adolescentes brasileiros. Rev. Saúde Pública, v. 50, p. 1-9, 2016. supl 1. Disponível em: https://scielosp.org/pdf/rsp/2016.v50suppl1/14s/pt. Acesso em: 22 abr. 2019. 
MARI, J. J.; JORGE, M. R. Epidemiologia dos transtornos mentais. In: MARI, J. J. et al. Guia de psiquiatria. Barueri, São Paulo: Manole, 2005, p. 1-5.

MARSH, E. J.; GRAHAM, S. A. Classificação e tratamento da psicopatologia infantil. In: CABAlLO, V. E., SIMÓN, M. A. (org.). Manual de psicologia clínica infantil e do adolescente - Transtornos Gerais. Tradução: Sandra M. Dolinsky. São Paulo: Livraria Santos Editora, 2005, p. 30-56.

MAY, R. O significado da ansiedade: as causas da integração e desintegração da personalidade. Tradução: Álvaro Cabral. Rio de Janeiro: Zahar Editores S.A., 1980.

ORGANIZAÇÃO MUNDIAL DE SAÚDE. Depression and other common mental disorders: global health estimates. Geneva: WHO; 2017. Disponível em: http://apps.who.int/iris/bitstream/10665/254610/1/WHO-MSD-MER-2017.2-eng.pdf. Acesso em: 20 maio 2019.

RAPPAPORT, C. R. Introdução. In: RAPPAPORT, C. R. (org.). Adolescência: abordagem psicanalítica. São Paulo: EPU, 1993, p. 1-23.

RIBEIRO, M. S.; POÇO, J. L. C. Identificando problemas e rastreando transtornos em atenção básica à saúde mental. In: RIBEIRO, M. S. (org.). Ferramentas para descomplicar a Atenção Básica em Saúde Mental. Juiz de Fora: Ed. UFJF, 2007, p. 27-52.

SADOCK, B. J.; SADOCK, V. A. Kaplan \& Sadock - Compêndio de psiquiatria: ciência do comportamento e psiquiatria clínica. Tradução: Cláudia Dornelles et al. 9. ed. Porto Alegre: Artmed, 2007.

THIENGO, D. L.; CAVALCANTE, M. T.; LOVISI, G. M. Prevalência de transtornos mentais entre crianças e adolescentes e fatores associados: uma revisão sistemática. J. bras. psiquiatr., Rio de Janeiro, v. 63, n. 4, p. 360-372, dez. 2014. Disponível em: http://www.scielo.br/scielo.php?script=sci_arttext\&pid=S0047-

20852014000400360\&lng=en\&nrm=iso. Acesso em: 29 maio 2019.

VIEIRA, M. A. et al. Saúde mental na escola. In: ESTANISLAU, G. M.; BRESSAN, R. A. (org.). Saúde Mental na escola: o que os educadores devem saber. Porto Alegre: Artmed, 2014, p. 13-23. 